\title{
The Level Set Method Of Joó And Its Use In Minimax Theory
}

\author{
J.B.G. Frenk and G. Kassay
}

\begin{tabular}{|l|l|}
\hline \multicolumn{2}{|l|}{ ERIM REPORT SERIES RESEARCH IN MANAGEMENT } \\
\hline ERIM Report Series reference number & ERS-2004-045-LIS \\
\hline Publication & June 2004 \\
\hline Number of pages & 10 \\
\hline Email address corresponding author & frenk@few.eur.nl \\
\hline Address & Erasmus Research Institute of Management (ERIM) \\
& Rotterdam School of Management / Rotterdam School of \\
& Economics \\
& Erasmus Universiteit Rotterdam \\
& P.O. Box 1738 \\
& 3000 DR Rotterdam, The Netherlands \\
& Phone: +31 10 408 1182 \\
& Fax: +31 10 408 9640 \\
& Email: info@erim.eur.nl \\
& Internet: www.erim.eur.nl \\
\hline
\end{tabular}

Bibliographic data and classifications of all the ERIM reports are also available on the ERIM website: www.erim.eur.nl 


\title{
ERASMUS RESEARCH INSTITUTE OF MANAGEMENT
}

\author{
REPORT SERIES \\ RESEARCH IN MANAGEMENT
}

\begin{tabular}{|c|c|c|}
\hline \multicolumn{3}{|c|}{ BIBLIOGRAPHIC DATA AND CLASSIFICATIONS } \\
\hline Abstract & \multicolumn{2}{|c|}{$\begin{array}{l}\text { In this paper we discuss the level set method of Joó and how to use it to give an elementary } \\
\text { proof of the well-known Sion's minimax result. Although this proof technique is initiated by Joó } \\
\text { and based on the inter-section of upper level sets and a clever use of the topological notion of } \\
\text { connectedness, it is not very well known and accessible for researchers in optimization. At the } \\
\text { same time we simplified the original proof of Joó and give a more elementary proof of the } \\
\text { celebrated Sion's minimax theorem. }\end{array}$} \\
\hline \multirow{3}{*}{$\begin{array}{l}\text { Library of Congress } \\
\text { Classification } \\
\text { (LCC) }\end{array}$} & $5001-6182$ & Business \\
\hline & $5201-5982$ & Business Science \\
\hline & HB 143.7 & Optimization Techniques \\
\hline \multirow{4}{*}{$\begin{array}{l}\text { Journal of Economic } \\
\text { Literature } \\
\text { (JEL) }\end{array}$} & M & Business Administration and Business Economics \\
\hline & M 11 & Production Management \\
\hline & R 4 & Transportation Systems \\
\hline & C 61 & Optimization Techniques ; Programming Models \\
\hline \multirow{4}{*}{$\begin{array}{l}\text { European Business Schools } \\
\text { Library Group } \\
\text { (EBSLG) }\end{array}$} & $85 \mathrm{~A}$ & Business General \\
\hline & $260 \mathrm{~K}$ & Logistics \\
\hline & $240 B$ & Information Systems Management \\
\hline & $255 B$ & Decision under constraints \\
\hline \multicolumn{3}{|c|}{ Gemeenschappelijke Onderwerpsontsluiting (GOO) } \\
\hline \multirow[t]{4}{*}{ Classification GOO } & 85.00 & Bedrijfskunde, Organisatiekunde: algemeen \\
\hline & 85.34 & Logistiek management \\
\hline & 85.20 & Bestuurlijke informatie, informatieverzorging \\
\hline & 31.43 & Functies van meerdere complexe variablen \\
\hline \multirow[t]{3}{*}{ Keywords GOO } & \multicolumn{2}{|c|}{ Bedrijfskunde / Bedrijfseconomie } \\
\hline & \multicolumn{2}{|c|}{ Bedrijfsprocessen, logistiek, management informatiesystemen } \\
\hline & \multicolumn{2}{|c|}{ Optimalisatie, minimax problemen } \\
\hline Free keywords & \multicolumn{2}{|c|}{ Level set method, minimax theorem, game theory } \\
\hline
\end{tabular}




\title{
The Level Set Method of Joó and its Use in Minimax Theory.
}

\author{
J.B.G.Frenk, G.Kassay
}

June 7, 2004

\begin{abstract}
In this paper we discuss the level set method of Joó and how to use it to give an elementary proof of the well-known Sion's minimax result. Although this proof technique is initiated by Joó and based on the intersection of upper level sets and a clever use of the topological notion of connectedness, it is not very well known and accessible for researchers in optimization. At the same time we simplified the original proof of Joó and give a more elementary proof of the celebrated Sion's minimax theorem.
\end{abstract}

\section{Introduction.}

Let $B$ and $A$ be nonempty topological spaces and consider the function $f$ : $A \times B \rightarrow \mathbb{R}$. In this note we are interested under which conditions the minimax inequality

$$
r_{*}:=\sup _{\mathbf{a} \in A} \inf _{\mathbf{b} \in B} f(\mathbf{a}, \mathbf{b})=\inf _{\mathbf{b} \in B} \sup _{\mathbf{a} \in A} f(\mathbf{a}, \mathbf{b}):=r^{*}
$$

holds. This equality plays a prominent role in game theory (cf.[13]) and duality theory in optimization (cf.[14], [6]). Actually we will give an elementary proof of the celebrated Sion's minimax theorem by using the level set method of Joó. In [8] a proof of Sion's minimax theorem is given using this approach and in this note we will simplify this proof.

\section{An elementary proof of Sion's minimax theorem avoid- ing the KKM lemma.}

For $f: A \times B \rightarrow \mathbb{R}$ a bifunction with $A$ and $B$ nonempty topological spaces we introduce for every $\mathbf{a} \in A$ and $\mathbf{b} \in B$ the related functions $G_{\mathbf{a}}: B \rightarrow \mathbb{R}$ 
and $F_{\mathbf{b}}: A \rightarrow \mathbb{R}$ given by

$$
G_{\mathbf{a}}(\mathbf{b})=F_{\mathbf{b}}(\mathbf{a}):=f(\mathbf{a}, \mathbf{b}) .
$$

In this note we are interested in proving Sion's minimax theorem by an elementary method and avoiding the less elementary KKM lemma used in the original proof given by Sion (cf.[12]). It is easy to see using the definition of $r^{*}$ and $r_{*}$ that always $r_{*} \leq r^{*}$ and so for $r^{*}=-\infty$ we obtain immediately $r_{*}=r^{*}$. Therefore we always assume that $r^{*}>-\infty$. To initiate the so-called level set method, introduced by Joó in [7], we introduce for every $r \in \mathbb{R}$ and $\mathbf{b} \in B$ the upper level set $U\left(F_{\mathbf{b}}, r\right) \subseteq A$ of the function $F_{\mathbf{b}}: A \rightarrow \mathbb{R}$, given by

$$
U\left(F_{\mathbf{b}}, r\right):=\left\{\mathbf{a} \in A: F_{\mathbf{b}}(\mathbf{a}) \geq r\right\} .
$$

The following result is shown in [7] and [8] and is the starting point of the so-called level set method.

Lemma 1 It follows that $r^{*}=r_{*}$ if and only if $\cap_{\mathbf{b} \in B} U\left(F_{\mathbf{b}}, r\right) \neq \emptyset$ for every $r<r^{*}$.

Proof. If $r^{*}=r_{*}>-\infty$, then for every $r<r^{*}=r_{*}$ there exists by the definition of $r_{*}$ some $\mathbf{a}_{0} \in A$ satisfying $\inf _{\mathbf{b} \in B} f\left(\mathbf{a}_{0}, \mathbf{b}\right)>r$. This shows that $\mathbf{a}_{0}$ belongs to the intersection $\cap_{\mathbf{b} \in B} U\left(F_{\mathbf{b}}, r\right)$ and so $\cap_{\mathbf{b} \in B} U\left(F_{\mathbf{b}}, r\right)$ is nonempty. To verify the reverse implication it is sufficient to verify that $r_{*} \geq$ $r^{*}$ or equivalently $r_{*}>r^{*}-\epsilon$ for every $\epsilon>0$. Consider now $r:=r^{*}-\epsilon$ for some $\epsilon>0$. By our assumption it follows that $\cap_{\mathbf{b} \in B} U\left(F_{\mathbf{b}}, r\right)$ is nonempty and so there exists some $\mathbf{a}_{0} \in A$ satisfying $\inf _{\mathbf{b} \in B} f\left(\mathbf{a}_{0}, \mathbf{b}\right) \geq r$. This implies $r_{*} \geq r$ and so the proof is completed.

By the above lemma we need to show that the intersection $\cap_{\mathbf{b} \in B} U\left(F_{\mathbf{b}}, r\right)$ is nonempty for every $r<r^{*}$. For arbitrary bifunctions $f$ this result does not hold and so we must impose some conditions on $f$. Before defining the proper class of bifunctions we introduce some facts well-known within topology. For $X$ a subset of a topological space with topology $\mathcal{F}$ and $S \subseteq X$ the set $S$ is called open in $X$ if there exists some set $O$ belonging to $\mathcal{F}$ with $S=X \cap O$. The topology generated in this way is called the relative topology induced by $X$ and with this topology the set $X$ is a topological space. We now introduce the well-known notion of connectedness (cf.[5]).

Definition 2 For any topological space $X$ a nonempty set $C \subseteq X$ is called connected, if for any two disjoint sets $C_{i}, i=1,2$ satisfying $C=C_{1} \cup C_{2}$ and $C_{i}, i=1,2$ open in $C$ it follows that $C_{1}$ or $C_{2}$ is empty. 
In the next definition we introduce the following class of functions already studied within optimization theory (cf.[9]). Observe in [9] the next class of functions is given a different definition, but as shown in Lemma 4 we are actually considering the same class of functions.

Definition 3 If $X$ is a topological space, the function $h: X \rightarrow \mathbb{R}$ is called connected if for every $\mathbf{x}_{1}, \mathbf{x}_{2} \in X$ there exists a connected set $C_{\mathbf{x}_{1} \mathbf{x}_{2}} \subseteq X$ containing $\mathbf{x}_{1}, \mathbf{x}_{2}$ such that

$$
h(\mathbf{x}) \leq \max \left\{h\left(\mathbf{x}_{1}\right), h\left(\mathbf{x}_{2}\right)\right\}
$$

for every $\mathbf{x} \in C_{\mathbf{x}_{1} \mathbf{x}_{2}}$.

If $X$ is a topological vector space (cf.[4]) the interval $\left[\mathbf{x}_{1}, \mathbf{x}_{2}\right]:=\left\{\alpha \mathbf{x}_{1}+\right.$ $\left.(1-\alpha) \mathbf{x}_{1}: 0 \leq \alpha \leq 1\right\}$ is connected in $X$ and so the above definition includes as a special case the set of quasiconvex functions (cf.[3]). As for quasiconvex functions it is easy to show the following result. Although the proof uses standard arguments from topology, we will list the proof for completeness.

Lemma 4 The function $h: X \rightarrow \mathbb{R}$ is connected if and only if for every $r \in \mathbb{R}$ the lower level set $L(h, r):=\{\mathbf{x} \in X: h(\mathbf{x}) \leq r\}$ is connected.

Proof. To show that the lower level sets are connected, consider some lower level set $L(h, r)$ with $\mathbf{x}_{1}$ belonging to $L(h, r)$ and let $\mathbf{x}_{2}$ be another arbitrary point belonging to $L(h, r)$. Since the function $h$ is connected there exists some connected set $C_{\mathbf{x}_{1} \mathbf{x}_{2}} \subseteq X$ containing $\mathbf{x}_{1}, \mathbf{x}_{2}$ such that

$$
h(\mathbf{x}) \leq \max \left\{h\left(\mathbf{x}_{1}\right), h\left(\mathbf{x}_{2}\right)\right\}
$$

for every $\mathbf{x}$ belonging to $C_{\mathbf{x}_{1} \mathbf{x}_{2}}$. This shows $C_{\mathbf{x}_{1} \mathbf{x}_{2}} \subseteq L(h, r)$ and since $\mathbf{x}_{2}$ is an arbitrary element of $L(h, r)$ we obtain

$$
\cup_{\mathbf{x}_{2} \in L(h, r)} C_{\mathbf{x}_{1} \mathbf{x}_{2}}=L(h, r) .
$$

By construction the intersection $\cap_{\mathbf{x}_{2} \in L(h, r)} C_{\mathbf{x}_{1} \mathbf{x}_{2}}$ contains the vector $\mathbf{x}_{1}$ and since for every $\mathbf{x}_{2} \in L(h, r)$ the set $C_{\mathbf{x}_{1} \mathbf{x}_{2}}$ is connected also $\cup_{\mathbf{x}_{2} \in L(h, c)} C_{\mathbf{x}_{1} \mathbf{x}_{2}}$ is connected (cf.[5]). Applying now relation (4) shows the desired result. To prove the reverse implication, consider some arbitrary $\mathbf{x}_{1}, \mathbf{x}_{2} \in X$ and introduce $r=\max \left\{h\left(\mathbf{x}_{1}\right), h\left(\mathbf{x}_{2}\right)\right\}$. Take now the set $C_{\mathbf{x}_{1} \mathbf{x}_{2}}$ equal to the connected set $L(h, c)$ and this set satisfies the desired property.

As already observed the above class of connected functions coincides with the class of the so-called LE-connected functions discussed by Martin (cf.[9]). 
Actually this class is more general than the class of functions studied by Avriel and Zang (cf.[2], [3]). In [2] and [3] a similar definition as done above is given with the additional condition that the set $C_{\mathbf{x}_{1} \mathbf{x}_{2}}$ is an arc-wise connected set. Finally, we like to mention that the class of connected functions with $C_{\mathbf{x}_{1} \mathbf{x}_{2}}$ is an arc-wise connected set were first introduced by [10] in an attempt to generalize the properties of quasiconvex functions. In order to prove our main lemma we also introduce the following class of functions.

Definition 5 If $X$ is a topological space, the collection of functions $h_{\gamma}: X \rightarrow$ $\mathbb{R}, \gamma \in \Gamma$ is called equiconnected if for every $\mathbf{x}_{1}, \mathbf{x}_{2} \in X$ there exists a connected set $C_{\mathbf{x}_{1} \mathbf{x}_{2}} \subseteq X$ containing $\mathbf{x}_{1}, \mathbf{x}_{2}$ such that

$$
h_{\gamma}(\mathbf{x}) \leq \max \left\{h_{\gamma}\left(\mathbf{x}_{1}\right), h_{\gamma}\left(\mathbf{x}_{2}\right)\right\}
$$

for every $\mathbf{x} \in C_{\mathbf{x}_{1} \mathbf{x}_{2}}$ and $\gamma \in \Gamma$.

If the set $X$ is a topological vector space and for every $\gamma \in \Gamma$ the function $f_{\gamma}$ is quasiconvex, then by taking $C_{\mathbf{x}_{1} \mathbf{x}_{2}}=\left[\mathbf{x}_{1}, \mathbf{x}_{2}\right]$ it follows immediately that the collection of functions $f_{\gamma}, \gamma \in \Gamma$ is equiconnected. Using now some properties of connectedness straightforward from the definition, the main result of this note is easy to show. The proof of this result is inspired by a similar proof as given in [8] with some nontrivial simplifications. Before mentioning this result we denote by $\mathcal{F}(B)$ the set of all finite subsets on $B$, while the function $F_{J}: A \rightarrow \mathbb{R}, J \in \mathcal{F}(B)$ is given by

$$
F_{J}(\mathbf{a}):=\min _{\mathbf{b} \in J} F_{\mathbf{b}}(\mathbf{a}) .
$$

Also recall that a function is called lower (upper) semicontinuous if its lower (upper) level sets are closed (cf.[1]).

Theorem 6 If the functions $F_{\mathbf{b}}, \mathbf{b} \in B$, respectively $G_{\mathbf{a}}, \mathbf{a} \in A$, are upper (lower) semicontinuous, the function $-F_{J}$ is connected for every $J$ belonging to $\mathcal{F}(B)$ and the collection of functions $G_{\mathbf{a}}, \mathbf{a} \in A$, is equiconnected, then for every $r<r^{*}$ and $J \in \mathcal{F}(B)$ the intersection $\cap_{\mathbf{b} \in J} U\left(F_{\mathbf{b}}, r\right)$ is nonempty.

Proof. If $J$ is a subset of $B$ consisting of one element, the result clearly holds by the definition of $r^{*}$ listed in relation (1). Suppose now for all sets $J$ belonging to $\mathcal{F}(B)$ and consisting of at most $k$ elements that

$$
\cap_{\mathbf{b} \in J} U\left(F_{\mathbf{b}}, r\right) \neq \emptyset
$$


for every $r<r^{*}$. To prove the result for all sets $J$ belonging to $\mathcal{F}(B)$ consisting of at most $k+1$ elements we assume by contradiction that there exists some set $J_{0}:=\left\{\mathbf{b}_{0}, \ldots, \mathbf{b}_{k}\right\} \subseteq B$ and some $r_{0}<r^{*}$ satisfying

$$
\cap_{i=0}^{k} U\left(F_{\mathbf{b}_{i}}, r_{0}\right)=\emptyset
$$

Since the collection of functions $\left(G_{\mathbf{a}}\right)_{\mathbf{a} \in A}$ is equiconnected, one can find for the points $\mathbf{b}_{0}, \mathbf{b}_{1}$ some connected set $C_{\mathbf{b}_{0} \mathbf{b}_{1}}$ containing $\mathbf{b}_{0}$ and $\mathbf{b}_{1}$ and satisfying

$$
f(\mathbf{a}, \mathbf{b}) \leq \max \left\{f\left(\mathbf{a}, \mathbf{b}_{0}\right), f\left(\mathbf{a}, \mathbf{b}_{1}\right)\right\}
$$

for every $\mathbf{b} \in C_{\mathbf{b}_{0} \mathbf{b}_{1}}$ and $\mathbf{a} \in A$. Introduce now the set valued mapping $\Phi_{r}$ : $C_{\mathbf{b}_{0} \mathbf{b}_{1}} \rightarrow 2^{A}$, given by

$$
\Phi_{r}(\mathbf{b}):=\left\{\mathbf{a} \in A: \min _{\beta \in\left\{\mathbf{b}_{2}, \ldots, \mathbf{b}_{k}, \mathbf{b}\right\}} F_{\beta}(\mathbf{a}) \geq r\right\}
$$

Since the function $\mathbf{a} \rightarrow-\min _{\beta \in\left\{\mathbf{b}_{2}, \ldots, \mathbf{b}_{k}, \mathbf{b}\right\}} F_{\beta}(\mathbf{a})$ is quasiconnected and the functions $F_{\mathbf{b}}$ are upper semicontinuous for every $\mathbf{b}$ it follows by relation (9) that the set $\Phi_{r}(\mathbf{b})$ is connected and closed for every $\mathbf{b}$ belonging to $C_{\mathbf{b}_{0} \mathbf{b}_{1}}$ and $r<r^{*}$. Moreover, by the induction hypothesis, listed in relation (6), we obtain that $\Phi_{r_{0}}(\mathbf{b})$ is nonempty for every $\mathbf{b} \in C_{\mathbf{b}_{0} \mathbf{b}_{1}}$ and by relations (8) and (9) that

$$
\Phi_{r_{0}}(\mathbf{b}) \subseteq \Phi_{r_{0}}\left(\mathbf{b}_{0}\right) \cup \Phi_{r_{0}}\left(\mathbf{b}_{1}\right)
$$

for every $\mathbf{b} \in C_{\mathbf{b}_{0} \mathbf{b}_{1}}$. Also by relations (7) and (9) it follows that $\Phi_{r_{0}}\left(\mathbf{b}_{0}\right) \cap$ $\Phi_{r_{0}}\left(\mathbf{b}_{1}\right)=\emptyset$ and so the nonempty sets

$$
S_{i}:=\left\{\mathbf{b} \in C_{\mathbf{b}_{0} \mathbf{b}_{1}}: \Phi_{r_{0}}(\mathbf{b}) \subseteq \Phi_{r_{0}}\left(\mathbf{b}_{i}\right)\right\}, i=0,1
$$

are disjoint and satisfy $S_{0} \cup S_{1} \subseteq C_{\mathbf{b}_{0} \mathbf{b}_{1}}$. To show that $S_{0} \cup S_{1}=C_{\mathbf{b}_{0} \mathbf{b}_{1}}$ consider for any $\mathbf{b} \in C_{\mathbf{b}_{0} \mathbf{b}_{1}}$ the closed sets

$$
A_{i}:=\Phi_{r_{0}}(\mathbf{b}) \cap \Phi_{r_{0}}\left(\mathbf{b}_{i}\right), i=0,1 .
$$

By relation (10) we obtain that $A_{1} \cup A_{2}=\Phi_{r_{0}}(\mathbf{b})$ and since $\Phi_{r_{0}}(\mathbf{b})$ is connected it must follow that $A_{0}$ or $A_{1}$ is empty. Hence by relation (10) either $\Phi_{r_{0}}(\mathbf{b}) \subseteq \Phi_{r_{0}}\left(\mathbf{b}_{1}\right)$ or $\Phi_{r_{0}}(\mathbf{b}) \subseteq \Phi_{r_{0}}\left(\mathbf{b}_{0}\right)$ and so the point $\mathbf{b}$ belongs to $S_{0} \cup S_{1}$. This means that the nonempty sets $S_{0}, S_{1}$ satisfy

$$
S_{0} \cup S_{1}=C_{\mathbf{b}_{0} \mathbf{b}_{1}} \text { and } S_{0} \cap S_{1}=\emptyset \text {. }
$$

We will now show that the sets $S_{0}$ and $S_{1}$ are open. Let $\mathbf{b}^{*}$ be an arbitrary element belonging to $S_{0}$. By our induction hypothesis we know that the set 
$\Phi_{r}\left(\mathbf{b}^{*}\right)$ is nonempty for every $r<r^{*}$ and this implies by the definition of $\Phi_{r}\left(\mathbf{b}^{*}\right)$ that

$$
\sup _{\mathbf{a} \in A} \inf _{\mathbf{b} \in\left\{\mathbf{b}_{2}, \ldots, \mathbf{b}_{k}, \mathbf{b}^{*}\right\}} f(\mathbf{a}, \mathbf{b}) \geq r
$$

for every $r<r^{*}$.This implies

$$
\sup _{\mathbf{a} \in A} \inf _{\mathbf{b} \in\left\{\mathbf{b}_{2}, \ldots, \mathbf{b}_{k}, \mathbf{b}^{*}\right\}} f(\mathbf{a}, \mathbf{b}) \geq r^{*}>r_{0}
$$

and so one can find some $\mathbf{a}_{0} \in \cap_{i=2}^{k} U\left(F_{\mathbf{b}_{i}}, r_{0}\right)$ satisfying

$$
f\left(\mathbf{a}_{0}, \mathbf{b}^{*}\right)>r_{0}
$$

By the lower semicontinuity of the function $\mathbf{b} \rightarrow f\left(\mathbf{a}_{0}, \mathbf{b}\right)$ and relation (13) there exists some neighborhood $\mathcal{U}\left(\mathbf{b}^{*}\right)$ of $\mathbf{b}^{*}$ such that $f\left(\mathbf{a}_{0}, \mathbf{b}\right)>r_{0}$ and so

$$
\mathbf{a}_{0} \in \Phi_{r_{0}}(\mathbf{b})
$$

for every $\mathbf{b}$ belonging to $\mathcal{U}\left(\mathbf{b}^{*}\right)$. Since $\mathbf{b}^{*} \in S_{0}$ and so $\Phi_{r_{0}}\left(\mathbf{b}^{*}\right) \subseteq \Phi_{r_{0}}\left(\mathbf{b}_{0}\right)$ it follows by relation (14) that

$$
\mathbf{a}_{0} \in \Phi_{r_{0}}(\mathbf{b}) \cap \Phi_{r_{0}}\left(\mathbf{b}_{0}\right)
$$

for every $\mathbf{b}$ belonging to $\mathcal{U}\left(\mathbf{b}^{*}\right) \cap C_{\mathbf{b}_{0} \mathbf{b}_{1}}$. This shows by relation (12) that $\Phi_{r_{0}}(\mathbf{b}) \subseteq \Phi_{r_{0}}\left(\mathbf{b}_{0}\right)$ for every $\mathbf{b}$ belonging to $\mathcal{U}\left(\mathbf{b}^{*}\right) \cap C_{\mathbf{b}_{0} \mathbf{b}_{1}}$ or equivalently

$$
\mathcal{U}\left(\mathbf{b}^{*}\right) \cap C_{\mathbf{b}_{0} \mathbf{b}_{1}} \subseteq S_{0} .
$$

Since $\mathbf{b}^{*} \in \mathcal{U}\left(\mathbf{b}^{*}\right) \cap C_{\mathbf{b}_{0} \mathbf{b}_{1}}$ is a set open in $C_{\mathbf{b}_{0} \mathbf{b}_{1}}$ and $\mathbf{b}^{*} \in S_{0}$ arbitrary this shows that

$$
S_{0}=C_{\mathbf{b}_{0} \mathbf{b}_{1}} \cap\left(\cup_{\mathbf{b}^{*} \in S_{0}} \mathcal{U}\left(\mathbf{b}^{*}\right)\right)
$$

and so the set $S_{0}$ is open in $C_{\mathbf{b}_{0} \mathbf{b}_{1}}$. Similarly one can verify that the set $S_{1}$ is open in $C_{\mathbf{b}_{0} \mathbf{b}_{1}}$ and by relation (12) and $C_{\mathbf{b}_{0} \mathbf{b}_{1}}$ connected it must follow that either $S_{0}$ or $S_{1}$ is empty. Since by relation (11) the point $\mathbf{b}_{i}$ belongs to $S_{i}, i=0,1$ this yields a contradiction and the proof is completed.

Applying now Lemma 1 we immediately deduce from Theorem 6 the following result.

Theorem 7 If the conditions of Theorem 6 hold and the set B is finite, then it follows that

$$
\min _{\mathbf{b} \in \mathbf{B}} \sup _{\mathbf{a} \in A} f(\mathbf{a}, \mathbf{b})=\sup _{\mathbf{a} \in A} \min _{\mathbf{b} \in \mathbf{B}} f(\mathbf{a}, \mathbf{b}) .
$$

Moreover, if $B$ is an infinite set, we obtain

$$
\inf _{\mathbf{b} \in B} \sup _{\mathbf{a} \in A} f(\mathbf{a}, \mathbf{b})=\inf _{J \in \mathcal{F}(B)} \sup _{\mathbf{a} \in A} \min _{\mathbf{b} \in J} f(\mathbf{a}, \mathbf{b}) .
$$


Proof. The first formula is an immediate consequence of Lemma 1 and Theorem 6 . To verify the second formula we observe that

$$
\inf _{\mathbf{b} \in B} \sup _{\mathbf{a} \in A} f(\mathbf{a}, \mathbf{b})=\inf _{J \in \mathcal{F}(B)} \min _{\mathbf{b} \in J} \sup _{\mathbf{a} \in A} f(\mathbf{a}, \mathbf{b}) .
$$

Applying now the first part yields the desired result.

To give a proof of Theorem 9 we first need to verify the following standard result.

Lemma 8 If the topological space $A$ is compact set and the functions $f_{\mathrm{b}}$ are upper semicontinuous for every $\mathbf{b} \in B$, then $\max _{\mathbf{a} \in A} \inf _{\mathbf{b} \in B} f(\mathbf{a}, \mathbf{b})$ is well defined and

$$
\inf _{J \in \mathcal{F}(B)} \sup _{\mathbf{a} \in A} \min _{\mathbf{b} \in J} f(\mathbf{a}, \mathbf{b})=\max _{\mathbf{a} \in A} \inf _{\mathbf{b} \in B} f(\mathbf{a}, \mathbf{b})
$$

Proof. Since the function $F_{\mathbf{b}}$ is upper semicontinuous for every $\mathbf{b} \in B$ we obtain that $p(\mathbf{a}):=\inf _{\mathbf{b} \in B} f(f(\mathbf{a}, \mathbf{b})$ is upper semicontinuous on $A$ and so by the Weierstrass-Lebesgue lemma (see Corollary 1.2 of [1]) and $A$ compact the function $p$ attains its maximum on $A$. This shows that $\max _{\mathbf{a} \in A} \inf _{\mathbf{b} \in B} f(\mathbf{a}, \mathbf{b})$ is well defined and to check the equality it is sufficient to verify that

$$
\alpha:=\max _{\mathbf{a} \in A} p(\mathbf{a}) \geq \inf _{J \in \mathcal{F}(B)} \max _{\mathbf{a} \in A} \min _{\mathbf{b} \in J} h(x, y):=\beta \text {. }
$$

If we assume by contradiction that $\alpha<\beta$ there exists some finite $\gamma$ satisfying $\alpha<\gamma<\beta$ and this implies by the definition of $\alpha$ that

$$
\cap_{\mathbf{b} \in B}\{\mathbf{a} \in A: f(\mathbf{a}, \mathbf{b}) \geq \gamma\}=\emptyset .
$$

Since $A$ is compact and $f_{\mathbf{b}}$ upper semicontinuous on $A$ for every $\mathbf{b} \in B$ we obtain that the set $\{\mathbf{a} \in A: f(\mathbf{a}, \mathbf{b}) \geq \gamma\}$ is compact for every $\mathbf{b} \in B$ and by relation (15) and the finite intersection property of compact sets (cf.[11]) we obtain for some $J_{0} \in \mathcal{F}(B)$ that

$$
\cap_{\mathbf{b} \in J_{0}}\{\mathbf{a} \in A: f(\mathbf{a}, \mathbf{b}) \geq \gamma\}=\emptyset .
$$

This implies $\min _{\mathbf{b} \in J_{0}} f(\mathbf{a}, \mathbf{b})<\gamma$ for every $\mathbf{a} \in A$ and by the first part $\max _{\mathbf{a} \in A} \inf _{\mathbf{b} \in B} f(\mathbf{a}, \mathbf{b})<\gamma<\beta$. This contradicts the definition of $\beta$ and so $\alpha \geq \beta$.

By Applying Lemma 8 and Theorem 7 one can show the following result, which contains as a special case Sion's minimax theorem. In Sion's minimax theorem it is additionally assumed that the sets $A$ and $B$ are convex, while the 
functions $G_{\mathbf{a}}$ are quasiconvex for every $\mathbf{a} \in A$ and $F_{\mathbf{b}}$ are quasiconcave for every $\mathbf{b} \in B$. This implies using Lemma 1 that the function $-F_{J}$ is connected for every $J$ belonging to $\mathcal{F}(B)$ and the collection of functions $G_{\mathbf{a}}, \mathbf{a} \in A$ is equiconnected.

Theorem 9 If the conditions of Theorem 6 hold and the set $A$ is a compact topological space, then it follows that

$$
\min _{\mathbf{b} \in B} \sup _{\mathbf{a} \in A} f(\mathbf{a}, \mathbf{b})=\sup _{\mathbf{a} \in A} \min _{\mathbf{b} \in B} f(\mathbf{a}, \mathbf{b}) .
$$

Proof. Since $A$ is compact and $F_{\mathbf{b}}$ is upper semicontinuous for every $\mathbf{b} \in B$ we obtain by Lemma 8 that

$$
\inf _{J \in \mathcal{F}(B)} \sup _{\mathbf{a} \in A} \min _{\mathbf{b} \in J} f(\mathbf{a}, \mathbf{b})=\max _{\mathbf{a} \in A} \inf _{\mathbf{b} \in B} f(\mathbf{a}, \mathbf{b})
$$

and applying Theorem 7 yields the desired result.

Actually by standard arguments one can slightly weaken the condition that $A$ is a compact topological space by replacing the compactness assumption by the condition that there exists some set $J \in \mathcal{F}(B)$ such that every upper level set of the function $F_{\mathbf{b}}$ is compact. Hence instead of assuming that $A$ is compact we assume that for every $r \in \mathbb{R}$ the set $\cap_{\mathbf{b} \in J}\{\mathbf{a} \in A: f(\mathbf{a}, \mathbf{b}) \geq r\}$ is compact for every $r \in \mathbb{R}$. This extension also covers as a special case duality results for convex programming problems with an interior point in its feasible region (Slater's condition).

\section{Conclusion.}

In this paper we have given an elementary proof of the important Sion's minimax result by means of the so-called level set method of Joó. Although a related proof already appeared in [8] we have simplified this proof and tried to make it accessible for operations researchers. As observed Sion minimax theorem can be used to prove the duality results in convex programming. From the proof of the main lemma it should be clear that the only properties of convex sets which are important in this proof are the observation that any intersection of convex sets is again convex and every convex set is connected. It also shows that Sion's minimax result is actually a topological result based on connectedness and so the duality results in convex optimization theory can be seen as topological results. 


\section{References}

[1] Aubin, J.B. Optima and Equilibra: An Introduction to Nonlinear Analysis, volume 140 of Graduate Texts in Mathematics. Springer Verlag, Berlin, 1993.

[2] Avriel, M. and Zang, I. Generalized arcwise-connected functions and characterizations of local-global minimum properties. Journal of Optimization Theory and Applications, 32. 4:407-425, 1980.

[3] Avriel, M., Diewert, W.E., Schaible, S. and Zang, I. Generalized Concavity, volume 36 of Mathematical Concepts and Methods in Engineering. Plenum Press, New York, 1970.

[4] Choquet, G. Lectures on Analysis Volume 1: Integration and Topological Vector Spaces. Mathematics Lecture Note Series. W.A.Benjamin, Reading, Massachusetts, 1976.

[5] Engelking, R. Outline of General Topology. North-Holland, Amsterdam, 1968.

[6] Faigle, U., Kern, W. and Stil, G. Algorithmic Principles of Mathematical Programming, volume 24 of Kluwer texts in Mathematical Sciences. Kluwer Academic Publishers, Dordrecht, 2002.

[7] Joó, I. Note on my paper: A simple proof of von Neumann's minimax theorem. Acta Math.Hung. 44, 3-4:363-365, 1984.

[8] Joó, I. On some convexities. Acta Math. Hung. 54, 1-2:163-172, 1989.

[9] Martin, D.H. Connected level sets, minimizing sets and uniqueness in optimization. Journal of Optimization Theory and Applications, 36. 1:7191, 1982.

[10] Ortega, J.M and Rheinbold, W.C. Iterative Solutions of Nonlinear Equations in Several Variables. Computer Science and Applied Mathematics. Academic Press, New York, 1970.

[11] Rudin, W. Principles of Mathematical Analysis. McGraw-Hill, New York, 1976.

[12] Sion, M. On general minimax theorems. Pacific J.Math, 8:171-176, 1958. 
[13] Vorob'ev, N.N. Game Theory: Lectures for Economists and Systems Scientists. Springer Verlag, New York, 1977.

[14] Walk, M. Theory of Duality in Mathematical Programming. SpringerVerlag, Wien, 1989. 


\section{Publications in the Report Series Research* in Management}

\section{ERIM Research Program: "Business Processes, Logistics and Information Systems"}

\section{4}

Smart Pricing: Linking Pricing Decisions with Operational Insights

Moritz Fleischmann, Joseph M. Hall and David F. Pyke

ERS-2004-001-LIS

http://hdl.handle.net/1765/1114

Mobile operators as banks or vice-versa? and: the challenges of Mobile channels for banks

L-F Pau

ERS-2004-015-LIS

http://hdl.handle.net/1765/1163

Simulation-based solution of stochastic mathematical programs with complementarity constraints: Sample-path analysis S. Ilker Birbil, Gül Gürkan and Ovidiu Listeş ERS-2004-016-LIS

http://hdl.handle.net/1765/1164

Combining economic and social goals in the design of production systems by using ergonomics standards Jan Dul, Henk de Vries, Sandra Verschoof, Wietske Eveleens and Albert Feilzer

ERS-2004-020-LIS

http://hdl.handle.net/1765/1200

Factory Gate Pricing: An Analysis of the Dutch Retail Distribution

H.M. le Blanc, F. Cruijssen, H.A. Fleuren, M.B.M. de Koster

ERS-2004-023-LIS

http://hdl.handle.net/1765/1443

A Review Of Design And Control Of Automated Guided Vehicle Systems

Tuan Le-Anh and M.B.M. De Koster

ERS-2004-030-LIS

http://hdl.handle.net/1765/1323

Online Dispatching Rules For Vehicle-Based Internal Transport Systems

Tuan Le-Anh and M.B.M. De Koster

ERS-2004-031-LIS

http://hdl.handle.net/1765/1324

Generalized Fractional Programming With User Interaction

S.I. Birbil, J.B.G. Frenk and S. Zhang

ERS-2004-033-LIS

http://hdl.handle.net/1765/1325

\footnotetext{
* A complete overview of the ERIM Report Series Research in Management: https://ep.eur.nl/handle/1765/1

ERIM Research Programs:

LIS Business Processes, Logistics and Information Systems

ORG Organizing for Performance

MKT Marketing

F\&A Finance and Accounting

STR Strategy and Entrepreneurship
} 
Meta-heuristics for dynamic lot sizing: A review and comparison of solution approaches

Raf Jans and Zeger Degraeve

ERS-2004-042-LIS

http://hdl.handle.net/1765/1336

A Multi-ltem Inventory Model With Joint Setup And Concave Production Costs

Z.P. Bayındır, S.I. Birbil and J.B.G. Frenk

ERS-2004-044-LIS

http://hdl.handle.net/1765/1535

The Level Set Method Of Joó And Its Use In Minimax Theory

J.B.G. Frenk and G. Kassay

ERS-2004-045-LIS

http://hdl.handle.net/1765/1537

Reinventing Crew Scheduling At Netherlands Railways

Erwin Abbink, Matteo Fischetti, Leo Kroon, Gerrit Timmer And Michiel Vromans

ERS-2004-046-LIS

http://hdl.handle.net/1765/1427

Intense Collaboration In Globally Distributed Teams: Evolving Patterns Of Dependencies And Coordination

Kuldeep Kumar, Paul C. van Fenema and Mary Ann Von Glinow

ERS-2004-052-LIS

http://hdl.handle.net/1765/1446

The Value Of Information In Reverse Logistics

Michael E. Ketzenberg, Erwin van der Laan and Ruud H. Teunter

ERS-2004-053-LIS

http://hdl.handle.net/1765/1447

Cargo Revenue Management: Bid-Prices For A 0-1 Multi Knapsack Problem

Kevin Pak and Rommert Dekker

ERS-2004-055-LIS

http://hdl.handle.net/1765/1449

Real-Time Scheduling Approaches For Vehicle-Based Internal Transport Systems

Tuan Le-Anh and M.B.M. De Koster

ERS-2004-056-LIS

http://hdl.handle.net/1765/1452

Activating Knowledge Through Electronic Collaboration: Vanquishing The Knowledge Paradox

S. Qureshi and P. Keen

ERS-2004-058-LIS

http://hdl.handle.net/1765/1473

A Grounded Theory Analysis Of E-Collaboration Effects For Distributed Project Management

S. Qureshi, M. Liu and D. Vogel

ERS-2004-059-LIS

http://hdl.handle.net/1765/1448

Collaborative Infrastructures For Mobilizing Intellectual Resources: Assessing Intellectual Bandwidth In A

Knowledge Intensive Organization

R. Verhoef and S. Qureshi

ERS-2004-060-LIS

http://hdl.handle.net/1765/1474

Satisfaction Attainment Theory As A Model For Value Creation

R.O. Briggs, S. Qureshi and B. Reining

ERS-2004-062-LIS

http://hdl.handle.net/1765/1450 
Diagnosis In The Olap Context

Emiel Caron, Hennie Daniels

ERS-2004-063-LIS

http://hdl.handle.net/1765/1492

A Deterministic Inventory/Production Model With General Inventory Cost Rate Function And Concave Production Costs Z.P. Bayındır, S.I. Birbil and J.B.G. Frenk

ERS-2004-064-LIS

http://hdl.handle.net/1765/1536 